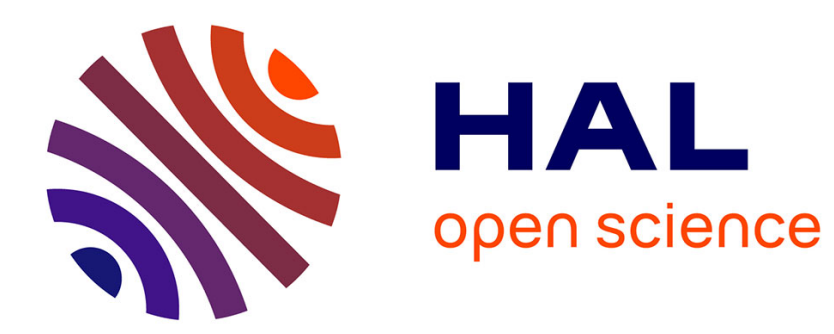

\title{
Competitive equilibrium under asymmetric information
}

Vathana Lyvath

\section{To cite this version:}

Vathana Lyvath. Competitive equilibrium under asymmetric information. 2007. hal-00019450v2

\section{HAL Id: hal-00019450 \\ https://hal.science/hal-00019450v2}

Preprint submitted on 9 Mar 2007

HAL is a multi-disciplinary open access archive for the deposit and dissemination of scientific research documents, whether they are published or not. The documents may come from teaching and research institutions in France or abroad, or from public or private research centers.
L'archive ouverte pluridisciplinaire HAL, est destinée au dépôt et à la diffusion de documents scientifiques de niveau recherche, publiés ou non, émanant des établissements d'enseignement et de recherche français ou étrangers, des laboratoires publics ou privés. 


\title{
Competitive market equilibrium under asymmetric information *
}

\author{
Vathana LY VATH \\ Laboratoire de Probabilités et \\ Modèles Aléatoires \\ CNRS, UMR 7599 \\ Université Paris 7 \\ e-mail: lyvath@math.jussieu.fr \\ February 2006 \\ this version February 2007
}

\begin{abstract}
This paper studies the existence of a competitive market equilibrium under asymmetric information. There are two agents involved in the trading of the risky assets: an "informed" trader and an "ordinary" trader. The market is competitive and the ordinary agent can infer the insider information from the risky assets price dynamics. The insider information is considered to be the total supply of the risky assets. The definition of market equilibrium is based on the law of supply-demand as described by a Rational Expectations Equilibrium of the Grossman and Stiglitz (1980) model. We show that equilibrium can be attained by linear dynamics of an admissible price process of the risky assets for a given linear supply dynamics.
\end{abstract}

Key words : insider trading, stochastic filtering theory, equilibrium, utility maximization.

JEL Classification : G10.

MSC Classification (2000) : 60H30.

*I would like to thank Huyên PHAM for helpful discussions and advises. 


\section{Introduction}

In recent years, financial mathematicians have been focusing on the model of asymmetric information. Asymmetric information arises when agents in the market do not have the same information filtration. They generally make an assumption regarding the extra information that is accessible uniquely by the "informed trader" or the "insider trader". This extra information could be, for example, the future liquidation price of the risky assets. Using the results of enlargement of filtration first developed by Jeulin (1980) and then Jacod (1985), many papers such as those of Grorud and Pontier (1998) and Amendinger et al (1998) focused on solving utility maximization problems in a security market where two investors have different information levels. In these papers, the security prices are assumed to evolve according to an exogenous diffusion. In Hillairet (2004), different types of asymmetric information, including "initial strong", "progressive strong" and "weak" information are studied. However, the drawback of the above models is that "ordinary" or "uninformed" agents cannot infer the insider information.

On the other hand, in Kyle (1985) and Back (1992), the market is competitive and the ordinary agents can obtain feedbacks from the market regarding the insider information. There have also been several other studies, published in the economic literature, on the impact of asymmetric information on stock price. The first such paper is the seminal paper of Grossman-Stiglitz (1980), followed by those of Glosten-Milgrom (1985). In Biais-Rochet (1997), we may find a very insightful survey of the literature on these areas, including those cited above. In Grossman-Stiglitz (1980), the agents are competitive and market is Walrasian, i.e. price equals supply and demand. The only exogenous part of this model may come from irrational traders, often called noise traders. In Biais-Rochet (1997), the objective is to analyse the price formation in a dynamic version of Grossman and Stiglitz model where stochastic control techniques can be used.

In the same framework, in our paper, we consider a financial market consisting of an "ordinary" agent, an "informed" agent and noise traders. While the ordinary agent can only observe the price dynamics of the risky assets, the "informed" agent has also access to the total supply of the risky assets. As in Back (1992), based on the observation of the price dynamics of the risky assets, "the ordinary" agent can infer the additional information of the "informed" agent. The purpose of the study is to see whether an equilibrium condition can be attained by linear dynamics of an admissible price process of the risky assets for a given linear supply dynamics. Like in the Grossman-Stiglitz model, the market is Walsarian, i.e. the agents involved in the market are competitive agents.

Our studies show that the existence of linear competitive market equilibrium under asymmetric information is directly related to the existence of solution to some associated nonlinear equations. Indeed, the equilibrium condition can be explicitly expressed in the form of a system of nonlinear equations. However, we may not determine whether the associated system of nonlinear equations leads to a nonempty set of solution. We nevertheless find that in the particular case where the total supply is a Brownian motion, the 
equilibrium can be reached and we explicitly obtain the linear dynamics of an admissible price process.

The plan of the paper is organized as follows. We define the model and the equilibrium condition in the second section, while in the third section, we use stochastic control techniques and filtering theory to solve agents' CARA optimization problem and then determine their optimal trading portfolio. In the fourth and fifth sections, we express the characterization of a potential equilibrium price and explicitly calculate the linear dynamics of an admissible price process in the particular case where the total supply dynamics is a Brownian motion.

\section{The model}

We consider a financial market with a risky stock and a risk-free bond. The risk-free interest rate is assumed to be zero. We are given a standard Brownian motion, $W=\left(W_{t}\right)_{t \in[0, T]}$ on a filtered probability space $\left(\Omega, \mathcal{F}, \mathbb{F}=\left(\mathcal{F}_{t}\right)_{t \in[0, T]}, \mathbb{P}\right)$ satisfying the usual conditions. $T$ is a fixed time at which all transactions are liquidated.

\subsection{Information and agents}

There are two rational competitive traders:

- The first one is an "informed" trader (insider trader), agent $I$, whose information is described by the filtration, $\mathbb{F}$, as he can observe both the risky assets price $S=\left(S_{t}\right)$ and the total supply of the risky assets $Z=\left(Z_{t}\right)$. He has a Constant Absolute Risk Aversion (CARA) with coefficient $\eta_{I}>0$, i.e. his utility function is equal to $U_{I}(v)=-\exp \left(-\eta_{I} v\right)$.

- The second trader is an ordinary economic agent, agent $O$, whose information is only given by the price observation. We denote by $\mathbb{F}^{O}$ the structure of his filtration. He also has a Constant Absolute Risk-Aversion (CARA) with coefficient $\eta_{O}>0$, i.e. his utility function is in the form : $U_{O}(v)=-\exp \left(-\eta_{O} v\right)$.

We assume that the supply $Z$ of the risky assets is a Gaussian process, governed by the s.d.e:

$$
d Z_{t}=\left(a(t) Z_{t}+b(t)\right) d t+\gamma(t) d W_{t}, \quad Z_{0}=z_{0} \in \mathbb{R},
$$

where $a, b$, and $\gamma$ are deterministic continuous functions from $[0, T]$ into $\mathbb{R}$.

\subsection{Admissible price function}

The purpose of this study is to find out whether an equilibrium condition can be attained by linear admissible price processes of the risky assets for a given linear supply dynamics as defined in (2.1). 
Definition 2.1 An admissible price process under $(\mathbb{P}, \mathbb{F})$ is a process in the form of:

$$
d S_{t}=S_{t}\left[\left(\alpha(t) Z_{t}+\beta(t)\right) d t+\sigma(t) d W_{t}\right], \quad 0 \leq t \leq T
$$

where $\alpha$ and $\beta$ are deterministic continuous functions from $[0, T]$ into $\mathbb{R}$, and $\sigma$ a deterministic continuous function from $[0, T]$ into $\mathbb{R}_{+}^{*}$.

We define $\mathcal{S}$ as the set of admissible price processes of risky assets.

The purpose is therefore to determine all set of functions $(\alpha, \beta, \sigma)$, i.e. admissible price processes, satisfying an equilibrium condition.

Remark 2.1 For a given $(\alpha, \beta, \sigma)$, the process $\left(Z_{t}, \ln \left(S_{t}\right)\right)_{t}$ is governed by a system of linear stochastic differential equations, which satisfies the usual conditions leading to the existence of a unique strong solution.

\subsection{Equilibrium}

Given an admissible price process $S$, a trading strategy for the "informed" agent (resp. the ordinary agent) is a $\mathbb{F}$ (resp. $\mathbb{F}^{O}$ )-predictable process $X$ integrable with respect to $S$. $X=\left(X_{t}\right)_{0 \leq t \leq T}$, where $X_{t}$ represents here the amount invested in the stocks at time $t$. We denote by $\mathcal{A}(\mathbb{F})\left(\right.$ resp. $\left.\mathcal{A}\left(\mathbb{F}^{O}\right)\right)$ this set of trading strategies, $X=\left(X_{t}\right)_{0 \leq t \leq T}$, which satisfy the integrability criteria:

$$
\int_{0}^{T}\left|X_{t}\right|^{2} d t<\infty, \quad \mathbb{P} \text { a.s. }
$$

Each rational agent's goal, with its own filtration, is to maximize his expected utility from terminal wealth. We now formulate the definition of market equilibrium based on the law of supply-demand as described by a Rational Expectation Equilibrium of the Grossman and Stiglitz model.

Definition 2.2 A market equilibrium is a pair $\left(\hat{X}^{I}, \hat{X}^{O}\right)$ and an element $\hat{S} \in \mathcal{S}$ such that :

(i) $\hat{X}^{I}$ is the solution of the insider agent's optimization problem :

$$
\max _{X \in \mathcal{A}(\mathbb{F})} \mathbb{E}\left[U_{I}\left(v_{I}+\int_{0}^{T} X_{t} \frac{d \hat{S}_{t}}{\hat{S}_{t}}\right)\right],
$$

where $v_{I} \in \mathbb{R}$ is the initial capital of the insider.

(ii) $\hat{X}^{O}$ is the solution of the ordinary agent's optimization problem :

$$
\max _{X \in \mathcal{A}\left(\mathbb{F}^{O}\right)} \mathbb{E}\left[U_{O}\left(v_{O}+\int_{0}^{T} X_{t} \frac{d \hat{S}_{t}}{\hat{S}_{t}}\right)\right],
$$

where $v_{O} \in \mathbb{R}$ is the initial capital of the ordinary agent.

(iii) the market clearing conditions hold:

$$
\hat{X}_{t}^{I}+\hat{X}_{t}^{O}=Z_{t}, \quad 0 \leq t \leq T .
$$

If $\left(\hat{X}^{I}, \hat{X}^{O}, \hat{S}\right)$ is a market equilibrium, then we say that $\hat{S}$ is an equilibrium pricing rule. 


\section{CARA utility maximization}

In this section, we determine the optimal trading portfolio of the ordinary and insider agents.

\section{1 "Informed" agent's optimization problem}

Given an admissible price process $S$, the self-financed wealth process of the investor with a trading portfolio $X \in \mathcal{A}(\mathbb{F})$ has a dynamics given by :

$$
\begin{aligned}
d V_{t} & =X_{t} \frac{d S_{t}}{S_{t}} \\
& =X_{t}\left[\alpha(t) Z_{t}+\beta(t)\right] d t+X_{t} \sigma(t) d W_{t} .
\end{aligned}
$$

The investor with initial wealth $v_{I}$ and constant risk aversion $\eta_{I}>0$ has to solve the optimization problem :

$$
\mathcal{J}_{I}\left(v_{I}\right)=\sup _{X \in \mathcal{A}(\mathbb{F})} \mathbb{E}\left[-\exp \left(-\eta_{I} V_{T}\right)\right]
$$

We consider the related dynamic optimization problem : for all $(t, v, z) \in[0, T] \times \mathbb{R} \times \mathbb{R}$,

$$
J_{I}(t, v, z)=\sup _{X \in \mathcal{A}(\mathbb{F})} \mathbb{E}\left[-\exp \left(-\eta_{I} V_{T}\right) \mid V_{t}=v, Z_{t}=z\right]
$$

so that

$$
\mathcal{J}_{I}\left(v_{I}\right)=J_{I}\left(0, v_{I}, z_{I}\right)
$$

The nonlinear dynamic programming equation associated to the stochastic control problem $(3.2)$ is :

$$
\frac{\partial J_{I}}{\partial t}(t, v, z)+\sup _{x \in \mathbb{R}} \mathcal{L}^{x} J_{I}(t, v, z)=0
$$

together with the terminal condition $J_{I}(T, v, z)=-\exp \left(-\eta_{I} v\right)$. Here $\mathcal{L}^{x}$ is the second order linear differential operator associated to the diffusion $(V, Z)$ for the constant control $X=x:$

$$
\begin{aligned}
\mathcal{L}^{x} J_{I}= & x[\alpha z+\beta] \frac{\partial J_{I}}{\partial v}+[a z+b] \frac{\partial J_{I}}{\partial z}+\frac{1}{2} x^{2} \sigma^{2} \frac{\partial^{2} J_{I}}{\partial v^{2}} \\
& +x \sigma \gamma \frac{\partial^{2} J_{I}}{\partial v \partial z}+\frac{1}{2} \gamma^{2} \frac{\partial^{2} J_{I}}{\partial z^{2}} .
\end{aligned}
$$

We make the logarithm transformation:

$$
J_{I}(t, v, z)=-\exp \left[-\eta_{I} v-\phi(t, z)\right] .
$$

Then the Bellman equation (3.3) becomes:

$$
\frac{\partial \phi}{\partial t}+\mathcal{L}_{Z} \phi+\sup _{x \in \mathbb{R}}\left[\eta_{I} x(\alpha z+\beta)-\frac{1}{2}\left|\eta_{I} x \sigma+\gamma \frac{\partial \phi}{\partial z}\right|^{2}\right]=0
$$


together with the terminal condition :

$$
\phi(T, z)=0
$$

Here $\mathcal{L}_{Z}$ is the second order linear operator associated to the diffusion $Z$ :

$$
\mathcal{L}_{Z} \phi=(a z+b) \frac{\partial \phi}{\partial z}+\frac{1}{2} \gamma^{2} \frac{\partial^{2} \phi}{\partial z^{2}} .
$$

The maximum in (3.4) is attained for :

$$
\hat{x}(t, z)=\frac{1}{\eta_{I} \sigma^{2}}\left[\alpha(t) z+\beta(t)-\sigma(t) \gamma(t) \frac{\partial \phi}{\partial z}(t, z)\right] .
$$

Substituting into (3.4) gives :

$$
\frac{\partial \phi}{\partial t}+\mathcal{L}_{Z} \phi+\frac{1}{2 \sigma^{2}}\left(\alpha z+\beta-\sigma \gamma \frac{\partial \phi}{\partial z}\right)^{2}-\frac{1}{2}\left(\gamma \frac{\partial \phi}{\partial z}\right)^{2}=0 .
$$

This is a linear second order equation for $\phi$. We are looking for a solution in the following form :

$$
\phi(t, z)=\frac{1}{2} P_{I}(t) z^{2}+Q_{I}(t) z+\chi_{I}(t)
$$

where $P_{I}, Q_{I}$, and $\chi_{I}$ are deterministic functions valued in $\mathbb{R}$. By substituting and cancelling quadratic terms in $z$, we see that (3.7) holds iff $P_{I}, Q_{I}$ and $\chi_{I}$ satisfy:

$$
\begin{aligned}
0 & =\dot{P}_{I}+2\left[a-\frac{\gamma \alpha}{\sigma}\right] P_{I}+\frac{\alpha^{2}}{\sigma^{2}} \\
P_{I}(T) & =0, \\
0 & =\dot{Q}_{I}+\left[a-\frac{\gamma \alpha}{\sigma}\right] Q_{I}+\frac{\beta}{\sigma^{2}}\left[\alpha-\gamma \sigma P_{I}\right]+b P_{I} \\
Q_{I}(T) & =0, \\
0 & =\dot{\chi}_{I}+\frac{\beta^{2}}{2 \sigma^{2}}+\left[b-\frac{\gamma \beta}{\sigma}\right] Q_{I}+\frac{1}{2} \gamma^{2} P_{I} \\
\chi_{I}(T) & =0 .
\end{aligned}
$$

By solving these differential equations, we obtain:

$$
\begin{aligned}
P_{I}(t)= & \exp \left[2 \int_{t}^{T}\left(a-\frac{\gamma \alpha}{\sigma}\right)(u) d u\right] \\
& \int_{t}^{T} \frac{\alpha^{2}}{\sigma^{2}}(s) e^{\left[-2 \int_{s}^{T}\left(a-\frac{\gamma \alpha}{\sigma}\right)(u) d u\right]} d s, \\
Q_{I}(t)= & \exp \left[\int_{t}^{T}\left(a-\frac{\gamma \alpha}{\sigma}\right)(u) d u\right] \\
& \int_{t}^{T}\left[\frac{\beta}{\sigma^{2}}\left(\alpha-P_{I} \gamma \sigma\right)+P_{I} b\right](s) e^{\left[\int_{s}^{T}-\left(a-\frac{\gamma \alpha}{\sigma}\right)(u) d u\right]} d s, \\
\chi_{I}(t)= & \int_{t}^{T}\left[\frac{\beta^{2}}{2 \sigma^{2}}+\left(b-\frac{a \beta}{\sigma}\right) Q_{I}+\frac{1}{2} \gamma^{2} P_{I}\right](u) d u .
\end{aligned}
$$


The main result of this section can then be stated as follows:

Theorem 3.1 The value function for problem (3.2) is equal to:

$$
J_{I}(t, v, z)=-\exp \left(-\eta_{I} v-\frac{1}{2} z^{2} P_{I}(t)-Q_{I}(t) z-\chi_{I}(t)\right)
$$

where $P_{I}, Q_{I}$ and $\chi_{I}$ are expressed in (3.11), (3.12), and (3.13). Moreover, the optimal trading portfolio for problem (3.1) is given by $\hat{X}_{t}^{I}=\hat{x}_{I}\left(t, Z_{t}\right), 0 \leq t \leq T$, where $\hat{x}_{I}(t, z)$ is defined on $[0, T] \times \mathbb{R}$ by :

$$
\begin{aligned}
\hat{x}_{I}(t, z) & =\Phi_{I}(t) z+H_{I}(t) \\
\Phi_{I}(t) & =\frac{1}{\eta_{I} \sigma^{2}(t)}\left[\alpha(t)-\sigma(t) \gamma(t) P_{I}(t)\right] \\
H_{I}(t) & =\frac{1}{\eta_{I} \sigma^{2}(t)}\left[\beta(t)-\sigma(t) \gamma(t) Q_{I}(t)\right] .
\end{aligned}
$$

Proof. See Appendix 1.

\subsection{Ordinary agent's optimization problem}

We now focus on the ordinary agent's optimization problem. The idea is to decompose the price process $\left(S_{t}\right)_{t}$ in its own filtration $\mathcal{F}_{t}^{O}$. We recall that the ordinary agent do not have access to the additional information, i.e. $\left(Z_{t}\right)_{0 \leq t \leq T}$, the total supply of the risky assets. However, he may obtain some feedbacks through the observations of the risky assets price process. Given his information filtration, $\mathbb{F}^{O}=\left(\mathcal{F}_{t}^{O}\right)_{t \in[0, T]}$, which is generated by the price process, $\mathcal{F}_{t}^{O}=\sigma\left(S_{s}, s \leq t\right)$, we may define the following process:

$$
\begin{aligned}
& \left\{\begin{array}{l}
\tilde{Z}_{t}=\mathbb{E}\left(Z_{t} \mid \mathcal{F}_{t}^{O}\right), \\
\tilde{Z}_{0}=\mathbb{E}\left[z_{0}\right],
\end{array}\right. \\
& \text { and }\left\{\begin{array}{l}
\Gamma(t)=\mathbb{E}\left[\left(Z_{t}-\tilde{Z}_{t}\right)^{2}\right], \\
\Gamma(0)=\operatorname{Var}\left(z_{0}\right),
\end{array}\right.
\end{aligned}
$$

where $\left(\tilde{Z}_{t}\right)_{t}$ represents the information obtained by the ordinary agent through the observations of the price process.

From Kalman Bucy filter results, Lipster and Shiryaev (2001) (Theorem 10.3) or Oksendal (2003), $\tilde{Z}_{t}$ and $\Gamma(t)$ are solution of the system of equations:

$$
\left\{\begin{array}{l}
d \tilde{Z}_{t}=\left[a(t) \tilde{Z}_{t}+b(t)\right] d t+\frac{1}{\sigma(t)}[\sigma(t) \gamma(t)+\alpha(t) \Gamma(t)] d W_{t}^{O} \\
\dot{\Gamma}(t)=2 a(t) \Gamma(t)-\frac{1}{\sigma^{2}(t)}[\gamma(t) \sigma(t)+\alpha(t) \Gamma(t)]^{2}+\gamma^{2}(t) \\
\tilde{Z}_{0}=\mathbb{E}\left[z_{0}\right], \quad \Gamma(0)=\operatorname{Var}\left(z_{0}\right)
\end{array}\right.
$$


where $W^{O}$ is a $\left(\mathbb{P}, \mathbb{F}^{O}\right)$-Brownian motion, the so-called innovation process.

We may obtain explicitly the expression of $\Gamma(t)$ by solving the Riccati equation (3.17) (see p4-7 in Reid (1972)):

$$
\Gamma(t)=\Gamma(0) \frac{\exp \left(-\int_{0}^{t}\left[-2 a(s)+2 \frac{\gamma(s) \alpha(s)}{\sigma(s)}\right] d s\right)}{1+\Gamma(0) \int_{0}^{t} \frac{\alpha^{2}(s)}{\sigma^{2}(s)} \exp \left(-\int_{0}^{s}\left[-2 a(u)+2 \frac{\gamma(u) \alpha(u)}{\sigma(u)}\right] d u\right) d s}
$$

We now need to decompose the price process $\left(S_{t}\right)_{t}$ in its own filtration $\mathcal{F}_{t}^{O}$. The dynamics of an admissible price process under $\left(\mathbb{P}, \mathbb{F}^{O}\right)$ is then given by :

$$
d S_{t}=S_{t}\left[\left(\alpha(t) \tilde{Z}_{t}+\beta(t)\right) d t+\sigma(t) d W_{t}^{O}\right] .
$$

The equivalent optimization problem for the ordinary agent with an initial wealth $v_{O}$ and constant risk aversion $\eta_{O}>0$ is :

$$
\mathcal{J}_{O}\left(v_{O}\right)=\sup _{X \in \mathcal{A}\left(\mathbb{F}^{O}\right)} \mathbb{E}\left[-\exp \left(-\eta_{O} V_{T}\right)\right]
$$

We consider the related dynamic optimization problem : for all $(t, v, z) \in[0, T] \times \mathbb{R} \times \mathbb{R}$,

$$
J_{O}(t, v, z)=\sup _{X \in A\left(\mathbb{F}^{O}\right)} \mathbb{E}\left[-\exp \left(-\eta_{O} V_{T}\right) \mid V_{t}=v, Z_{t}=z\right]
$$

so that $\mathcal{J}_{O}\left(v_{O}\right)=J_{O}\left(0, v_{O}, z_{O}\right)$.

Using the same arguments as in Theorem 3.1, we obtain the following results for ordinary agent:

Theorem 3.2 The optimal trading portfolio for problem (3.19) is given by $\hat{X}_{t}^{O}=\hat{x}_{O}\left(t, Z_{t}\right)$, $0 \leq t \leq T$, where $\hat{x}_{O}(t, z)$ is defined on $[0, T] \times \mathbb{R}$ by :

$$
\begin{aligned}
\hat{x}_{O}(t, z) & =\Phi_{O}(t) z+H_{O}(t), \\
\Phi_{O}(t) & =\frac{1}{\eta_{O} \sigma^{2}(t)}\left[\alpha(t)-\sigma(t) \bar{\gamma}(t) P_{O}(t)\right], \\
H_{O}(t) & =\frac{1}{\eta_{O} \sigma^{2}(t)}\left[\beta(t)-\sigma(t) \bar{\gamma}(t) Q_{O}(t)\right],
\end{aligned}
$$

and $P_{O}$ and $Q_{O}$ are expressed as:

$$
\begin{aligned}
P_{O}(t)= & \exp \left[2 \int_{t}^{T}\left(a-\frac{\bar{\gamma} \alpha}{\sigma}\right)(s) d s\right] \\
& \int_{t}^{T} \frac{\alpha^{2}}{\sigma^{2}}(s) \exp \left[-2 \int_{s}^{T}\left(a-\frac{\bar{\gamma} \alpha}{\sigma}\right)(u) d u\right] d s \\
Q_{O}(t)= & \exp \left[\int_{t}^{T}\left(a-\frac{\bar{\gamma} \alpha}{\sigma}\right)(s) d s\right] \int_{t}^{T}\left[\frac{\beta}{\sigma^{2}}\left[\alpha-P_{O} \bar{\gamma} \sigma\right]+P_{O} b\right](s) \\
& \exp \left[-\int_{s}^{T}\left(a-\frac{\bar{\gamma} \alpha}{\sigma}\right)(u) d u\right] d s
\end{aligned}
$$


with

$$
\bar{\gamma}=\frac{1}{\sigma}[\sigma \gamma+\alpha \Gamma]
$$

Remark 3.1 $P_{O}$ and $Q_{O}$ satisfy

$$
\begin{aligned}
0 & =\dot{P}_{O}+2\left[a-\frac{\bar{\gamma} \alpha}{\sigma}\right] P_{O}+\frac{\alpha^{2}}{\sigma^{2}} \\
P_{O}(T) & =0, \\
0 & =\dot{Q}_{O}+\left[a-\frac{\bar{\gamma} \alpha}{\sigma}\right] Q_{O}+\frac{\beta}{\sigma^{2}}\left[\alpha-\bar{\gamma} \sigma P_{O}\right]+b P_{O} \\
Q_{O}(T) & =0 .
\end{aligned}
$$

\section{Characterization of the equilibrium price}

In this section, we give a characterization of a market equilibrium as defined in Definition 2.2. Using the optimal strategy of each agent determined in the previous section, we find that the equilibrium condition can be explicitly expressed as a nonlinear system.

Theorem 4.1 The equilibrium condition is equivalent to the following nonlinear system of at most three equations with three unknown variables, $\alpha, \beta$, and $\sigma$ :

$$
\begin{cases}\frac{1}{\eta_{O}}\left[\beta(t)-\sigma(t) \bar{\gamma}(t) Q_{O}(t)\right]+\frac{1}{\eta_{I}}\left[\beta(t)-\sigma(t) \gamma(t) Q_{I}(t)\right] & =0 \\ \frac{1}{\eta_{I} \sigma^{2}(t)}\left[\alpha(t)-\sigma(t) \gamma(t) P_{I}(t)\right]-1 & =0 \\ {\left[\alpha(t)-\sigma(t) \bar{\gamma}(t) P_{O}(t)\right] \operatorname{Var}\left(\tilde{Z}_{t}\right)} & =0 .\end{cases}
$$

Proof. The equilibrium pricing rule is given by

$$
\hat{X}_{I}\left(t, Z_{t}\right)+\hat{X}_{O}\left(t, \tilde{Z}_{t}\right)=Z_{t} .
$$

To simplify the calculations, we assume, without loss of generality, that the Gaussian process $\left(Z_{t}-\tilde{Z}_{t}, \tilde{Z}_{t}\right)$ is centered. The equilibrium (4.2) is equivalent to

$$
\left\{\begin{array}{lll}
\mathbb{E}\left[\hat{X}_{I}\left(t, Z_{t}\right)+\hat{X}_{O}\left(t, \tilde{Z}_{t}\right)\right] & =\mathbb{E}\left[Z_{t}\right] \\
\operatorname{Var}\left[\hat{X}_{I}\left(t, Z_{t}\right)+\hat{X}_{O}\left(t, \tilde{Z}_{t}\right)\right] & = & \operatorname{Var}\left(Z_{t}\right)
\end{array}\right.
$$

Using (3.14) and (3.21), the equilibrium condition becomes:

$$
\begin{cases}H_{O}(t)+H_{I}(t) & =0 \\ \left(\Phi_{I}(t)-1\right)\left(Z_{t}-\tilde{Z}_{t}\right)+\left(\Phi_{O}(t)+\Phi_{I}(t)-1\right) \tilde{Z}_{t} & =0\end{cases}
$$

Multiplying the latter by $\tilde{Z}_{t}$ and taking its expectation, we obtain:

$$
\left(\Phi_{I}(t)-1\right) \mathbb{E}\left[\left(Z_{t}-\tilde{Z}_{t}\right) \tilde{Z}_{t}\right]+\left(\Phi_{O}(t)+\Phi_{I}(t)-1\right) \operatorname{Var}\left(\tilde{Z}_{t}\right)=0 .
$$


Using the the fact that

$$
\left.\mathbb{E}\left[\left(Z_{t}-\tilde{Z}_{t}\right) \tilde{Z}_{t}\right]=\mathbb{E}\left[\left(Z_{t} \tilde{Z}_{t}-\tilde{Z}_{t}^{2}\right)\right]=\mathbb{E}\left[\tilde{Z}_{t} \mathbb{E}\left[Z_{t} \mid \mathcal{F}_{t}^{O}\right]-\tilde{Z}_{t}^{2}\right)\right]=0,
$$

and plugging into (4.5), we have:

$$
\left(\Phi_{I}(t)+\Phi_{O}(t)-1\right) \operatorname{Var}\left(\tilde{Z}_{t}\right)=0 .
$$

Plugging this latter equation into the second equation of (4.4), we obtain:

$$
\left(\Phi_{I}(t)-1\right)\left(Z_{t}-\tilde{Z}_{t}\right)=0
$$

As such, the equilibrium condition becomes:

$$
\left\{\begin{array}{lll}
H_{O}(t)+H_{I}(t) & = & 0 \\
\left(\Phi_{I}(t)-1\right) \Gamma(t) & = & 0 \\
\left(\Phi_{I}(t)+\Phi_{O}(t)-1\right) \operatorname{Var}\left(\tilde{Z}_{t}\right) & = & 0
\end{array}\right.
$$

Since $\Gamma(t)>0$, the above equilibrium condition is also written as:

$$
\begin{cases}H_{O}(t)+H_{I}(t) & =0 \\ \Phi_{I}(t)-1 & =0 \\ \Phi_{O}(t) \operatorname{Var}\left(\tilde{Z}_{t}\right) & =0\end{cases}
$$

and the required results are obtained by substituting the expression of $H_{O}, H_{I}, \Phi_{O}$, and $\Phi_{I}$.

Remark 4.1 While the explicit expression of the equilibrium condition is in the form of a nonlinear system, we do not know whether this system leads to a nonempty set of solution. Recall that $Q_{O}, Q_{I}, P_{O}, P_{I}$, and $\bar{\gamma}$ are dependent on the unknown variables $\alpha, \beta$, and $\sigma$, see (3.11), (3.12), (3.24), and (3.25).

Remark 4.2 In the case of a non-degenerated model, i.e. $\tilde{Z} \neq 0$, the equilibrium is equivalent to the following system:

$$
\begin{cases}\frac{1}{\eta_{O}}\left[\beta(t)-\sigma(t) \bar{\gamma}(t) Q_{O}(t)\right]+\frac{1}{\eta_{I}}\left[\beta(t)-\sigma(t) \gamma(t) Q_{I}(t)\right] & =0 \\ \frac{1}{\eta_{I} \sigma^{2}(t)}\left[\alpha(t)-\sigma(t) \gamma(t) P_{I}(t)\right]-1 & =0 \\ \alpha(t)-\sigma(t) \bar{\gamma}(t) P_{O}(t) & =0\end{cases}
$$

\section{Equilibrium in the case: $Z_{t}=W_{t}$}

We take the particular case of $Z_{t}=W_{t}$, i.e. $a(t)=b(t)=0$ and $\gamma(t)=1$.

Proposition 5.1 In the case of $Z_{t}=W_{t}$, the equilibrium is reached and the linear dynamics of an admissible price process is given by

$$
d S_{t}=S_{t}\left[\alpha(t) Z_{t} d t+\sigma(t) d W_{t}\right]
$$


with

$$
\begin{aligned}
\sigma(t) & =\frac{1}{\eta_{I}}\left[\mu(t)+\frac{1}{3} \frac{\mu^{2}(t)}{\mu_{T}}\left(1-\frac{\mu^{3}(t)}{\mu_{T}^{3}}\right)\right], \\
\alpha(t) & =\sigma(t) \mu(t),
\end{aligned}
$$

where

$$
\mu(t)=\frac{\mu_{T}}{1+\mu_{T}(T-t)} \quad \text { and } \mu_{T} \text { is any arbitrary positive constant. }
$$

Remark 5.3 The equilibrium condition does not depend on the

CARA coefficient of the ordinary agent. In economic sense, this means that the "informed" agent defines his trading strategy in order to maximize his expected utility from terminal wealth and imposes his optimal trading strategy upon the ordinary trader.

Proof of proposition 5.1. Let us set $\mu(t)=\frac{\alpha(t)}{\sigma(t)}$. From (3.26), (3.17), and (3.27), we obtain :

$$
\left\{\begin{array}{l}
\bar{\gamma}(t)=1+\mu(t) \Gamma(t) \\
\dot{\Gamma}(t)=1-[1+\mu(t) \Gamma(t)]^{2}, \\
\dot{P_{O}}=-\mu(t)^{2}+2 \mu(t)[1+\mu(t) \Gamma(t)] P_{O}(t) .
\end{array}\right.
$$

While the first relation in (3.17) becomes:

$$
d \tilde{Z}_{t}=[1+\mu(t) \Gamma(t)] d W_{t}^{O} .
$$

Thus

$$
\operatorname{Var}\left(\tilde{Z}_{t}\right)=\int_{0}^{t}[1+\mu(s) \Gamma(s)]^{2} d s
$$

The equilibrium pricing rule (4.1) becomes :

$$
\begin{aligned}
\frac{1}{\eta_{O}}\left[\beta(t)-\sigma(t)(1+\mu(t) \Gamma(t)) Q_{O}(t)\right] & \\
+\frac{1}{\eta_{I}}\left[\beta(t)-\sigma(t) Q_{I}(t)\right] & =0, \\
\frac{1}{\eta_{I} \sigma(t)}\left[\mu(t)-P_{I}(t)\right]-1 & =0, \\
{\left[\mu(t)-(1+\mu(t) \Gamma(t)) P_{O}(t)\right] \operatorname{Var}\left(\tilde{Z}_{t}\right) } & =0 .
\end{aligned}
$$

The latter relation (5.17) is equivalent to, for all $t \in[0, T]$ :

$$
\mu(t)-(1+\mu(t) \Gamma(t)) P_{O}(t)=0
$$

or

$$
\operatorname{Var}\left(\tilde{Z}_{t}\right)=0
$$

We show that the degenerated case (5.19) cannot happen. Assume that there exists $t$ such that the latter equation (5.19) is satisfied, then by using (5.14), we have

$$
1+\mu(s) \Gamma(s)=0, \forall s \in[0, t] .
$$


As $\Gamma_{s}=s$, we obtain:

$$
\mu(s)=-\frac{1}{s}, \quad \forall s \in[0, t] .
$$

We recall that $\mu=\frac{\alpha}{\sigma}$, as such, we either have $\lim _{s \rightarrow 0} \alpha(s)=\infty$ or $\lim _{s \rightarrow 0} \sigma(s)=0$, leading to a non admissible price function (see Remark 2.1).

As such, relation (5.17) is equivalent to

$$
\mu(t)-(1+\mu(t) \Gamma(t)) P_{O}(t)=0
$$

By deriving the latter equation, we obtain:

$$
\dot{\mu}-(1+\mu \Gamma) \dot{P}_{O}-(\dot{\mu} \Gamma+\mu \dot{\Gamma}) P_{O}=0
$$

Using the expressions of $\dot{\Gamma}$ and $\dot{P}_{O}$ in $(5.12)$, we obtain the following equation :

$$
\begin{aligned}
\dot{\mu}-(1+\mu \Gamma)\left[-\mu(t)^{2}+2 \mu(1+\mu \Gamma) P_{O}\right] & \\
-\left[\dot{\mu} \Gamma+\mu\left(1-[1+\mu(t) \Gamma]^{2}\right)\right] P_{O} & =0 .
\end{aligned}
$$

A straightforward simplification gives us:

$$
\dot{\mu}\left(1-\Gamma P_{O}\right)+\mu^{2}(1+\mu(t) \Gamma)-\mu^{2} P_{O} \Gamma(2+\mu \Gamma)-2 \mu P_{O}=0
$$

Using (5.21), we obtain :

$$
\dot{\mu}\left(1-\Gamma P_{O}\right)-\mu P_{O}=0 .
$$

Using once more (5.21), we obtain the following equation for $\mu$ :

$$
\dot{\mu}(t)=\mu^{2}(t), t \in[0, T] .
$$

As such,

$$
\mu(t)=\mu_{T} \frac{1}{1+\mu_{T}(T-t)}
$$

which raises no problem of definition in the case of $\mu_{T}>0$.

From equation (5.15) and (5.16), we obtain the explicit expression of $\sigma$ and $\beta$, and therefore $\alpha$.

$$
\begin{aligned}
\sigma(t) & =\frac{1}{\eta_{I}}\left[\mu(t)+\frac{1}{3} \frac{\mu^{2}(t)}{\mu_{T}}\left(1-\frac{\mu^{3}(t)}{\mu_{T}^{3}}\right)\right] \\
\alpha(t) & =\sigma(t) \mu(t) \\
\beta(t) & =0 \\
\text { where } \mu(t) & =\frac{\mu_{T}}{1+\mu_{T}(T-t)} .
\end{aligned}
$$

We check that when $\mu_{T}>0, \mu$ and $\sigma$ are positive for $t \in[0, T]$. 


\section{Appendix: Proof of Theorem 3.1}

We set:

$$
\begin{aligned}
\phi(t, z) & =\frac{1}{2} P_{I}(t) z^{2}+Q_{I}(t) z+\chi_{I}(t) \\
g(t, v, z) & =-\eta_{I} v-\phi(t, z)
\end{aligned}
$$

Where $P_{I}, Q_{I}$, and $\chi_{I}$ are expressed as above $[$ see $(3.11),(3.12),(3.13)]$

By differentiating, we obtain:

$$
\begin{array}{r}
\frac{\partial g}{\partial t}=-\frac{\partial \phi}{\partial t}, \frac{\partial g}{\partial v}=-\eta_{I}, \frac{\partial g}{\partial z}=-\frac{\partial \phi}{\partial z} \\
\mathcal{L}^{x} g=-\eta_{I}(\alpha z+\beta) x-\mathcal{L}_{Z} \phi
\end{array}
$$

By applying Itô's formula to $g\left(t, V_{t}, Z_{t}\right)$ for any $X \in \mathcal{A}(\mathbb{F})$ between $t$ and $T$, we obtain :

$$
\begin{aligned}
g\left(T, V_{T}, Z_{T}\right)= & g\left(t, V_{t}, Z_{t}\right)+\int_{t}^{T}\left(\frac{\partial g}{\partial t}+\mathcal{L}^{X_{u}} g\right)\left(u, V_{u}, Z_{u}\right) d u \\
& +\int_{t}^{T}\left(\frac{\partial g}{\partial v} X \sigma+\left(\frac{\partial g}{\partial z}\right) \gamma\right)\left(u, V_{u}, Z_{u}\right) d W_{u} \\
= & g\left(t, V_{t}, Z_{t}\right) \\
& +\int_{t}^{T}\left(-\frac{\partial \phi}{\partial t}-\mathcal{L}_{Y} \phi-\eta_{I} X(\alpha Z+\beta)\right)\left(u, Z_{u}\right) d u \\
& +\int_{t}^{T}\left(-\eta_{I} X_{u} \sigma(u)-\left(\frac{\partial \phi}{\partial z}\right)\left(u, Z_{u}\right) \gamma(u)\right) d W_{u} \\
= & g\left(t, V_{t}, Z_{t}\right)-\int_{t}^{T}\left(\frac{\partial \phi}{\partial t}+\mathcal{L}_{Y} \phi+\eta_{I} X_{u}(\alpha Z+\beta)\right. \\
& \left.-\left.\frac{1}{2}\right|_{I} X \sigma+\left.\gamma \frac{\partial \phi}{\partial z}\right|^{2}\right)\left(u, Z_{u}\right) d u \\
& -\int_{t}^{T}\left(\eta_{I} X_{u} \sigma(u)+\left(\frac{\partial \phi}{\partial z}\right)\left(u, Z_{u}\right) \gamma(u)\right) d W_{u} \\
& -\frac{1}{2} \int_{t}^{T}\left|\eta_{I} X \sigma+\gamma \frac{\partial \phi}{\partial z}\right|^{2}\left(u, Z_{u}\right) d u .
\end{aligned}
$$

We now consider the exponential local $(\mathbb{P}, \mathbb{F})$-martingale for any $X \in \mathcal{A}(\mathbb{F})$ :

$$
\begin{aligned}
\xi_{t}^{X}= & \exp \left\{-\int_{t}^{T}\left(\eta_{I} X_{u} \sigma(u)+\left(\frac{\partial \phi}{\partial z}\right)\left(u, Z_{u}\right) \gamma(u)\right) d W_{u}\right. \\
& \left.-\frac{1}{2} \int_{t}^{T}\left|\eta_{I} X \sigma+\gamma \frac{\partial \phi}{\partial z}\right|^{2}\left(u, Z_{u}\right) d u .\right\}
\end{aligned}
$$

From PDE (3.4) satisfied by $\phi$, relation (A.1) yields for all $X \in \mathcal{A}(\mathbb{F})$ :

$$
\exp \left(g\left(T, V_{t}, Z_{T}\right)\right) \geq \exp \left(g\left(t, V_{t}, Z_{t}\right)\right) \cdot \frac{\xi_{T}^{X}}{\xi_{t}^{X}} .
$$


Since $g(T, v, z)=-\eta_{I} v$ and $\xi^{X}$ is a $(\mathbb{P}, \mathbb{F})$-supermartingale, we obtain by taking conditional expectation in the previous inequality :

$$
\mathbb{E}\left[-\exp \left(-\eta_{I} V_{T}\right) \mid V_{t}=v, Y_{t}=y\right] \leq-\exp (g(t, v, z)),
$$

for all $X \in \mathcal{A}(\mathbb{F})$ and so :

$$
J_{\mathbb{F}}(t, v, z) \leq-\exp (g(t, v, z))
$$

Consider now the control strategy $\hat{X}_{t}=\hat{x}\left(t, Z_{t}\right), 0 \leq t \leq T$, where $\hat{x}$ is defined in (3.6) or more explicitly in (3.14). Then, we clearly have $\hat{X} \in \mathcal{A}(\mathbb{F})$, and we have now equality in (A.2) since $\hat{x}$ attains the supremum in the $\mathrm{PDE}(3.4)$ :

$$
\exp \left(g\left(T, V_{t}, Z_{T}\right)\right)=\exp \left(g\left(t, V_{t}, Z_{t}\right)\right) \cdot \frac{\xi_{T}^{\hat{X}}}{\xi_{t}^{\hat{X}}} .
$$

Observe that :

$$
\begin{aligned}
& \eta_{I} \hat{X}_{u} \sigma(u)+\left(\frac{\partial \phi}{\partial z}\right)\left(u, Z_{u}\right) \gamma(u)=Z_{u}\left(\eta_{I} \Phi(u) \sigma(u)+P_{I}(u) \gamma(u)\right)+ \\
& \eta_{I} H(u) \sigma(u)+Q_{I}(u) \gamma(u) \text {. }
\end{aligned}
$$

Since $Z$ is a Gaussian process, it follows that for some $\delta>0$, we have :

$$
\mathbb{E}\left[\exp \left(\delta\left|\eta_{I} X_{u} \sigma+\gamma \frac{\partial \phi}{\partial z}\right|^{2}\left(u, Z_{u}\right)\right)\right]<\infty
$$

Therefore by Liptser, Shiryaev $\left(1977\right.$, p.220), $\xi^{\hat{X}}$ is a $(\mathbb{P}, \mathbb{F})$-martingale and so by taking conditional expectation in (A.4), we have :

$$
\mathbb{E}\left[-\exp \left(-\eta_{I} V_{T}\right) \mid V_{t}=v, Z_{t}=z\right]=-\exp (g(t, v, z)),
$$

for the wealth process $V$ controlled by the trading portfolio $\hat{X}$. This last equality combined with (A.3) ends the proof.

\section{References}

[1] Amendinger, J., Imkeller, P., and Schweizer, M. (1998): Additional logarithmic utility of an insider. Stoch. Proc. Appl., 75, 263-286.

[2] Back, K. (1992): Insider trading in continuous time. Review of Financial Studies 5, 387-409.

[3] Biais, B., Rochet, J.C. (1997), Risk Sharing, adverse selection and market structure. Financial Mathematics, Lectures Notes on Mathematics, Springer Verlag.

[4] Glosten, L.R., Milgrom, P.R.(1985): Bid, Ask and Transaction Prices in a Specialist Market with Heterogeneously Informed Traders. Journal of Financial Economics 14, 71-100. 
[5] Grorud, A., Pontier, M. (1998): Insider trading in a continuous time market model. International Journal of Theoritical and Applied Finance 1, 331-347.

[6] Grossman, S.J., Stiglitz, J.E. (1980): On the Impossibility of Informationally Efficient Markets. American Economic Review 70, 393-408.

[7] Hillairet, C. (2004): Existence of an equilibrium on a financial market with different asymmetric information. preprint, LSP.

[8] Jacod, J. (1985) : Grossissement Initial, Hypothèse (H') et Théorème de Girsanov. Lecture Notes 118, Springer-Verlag, 15-35.

[9] Jeulin, T. (1980): Semi-martingales et grossissement d'une filtration. Lecture Notes Math 833, Springer-Verlag.

[10] Kyle, A.S. (1985): Continuous Auctions and Insider Trading. Econometrica 53, 13351355 .

[11] Lipster, R. Sh., Shiryaev, A. N. (2001), Statistics of Random Processes. I, New York, Springer-Verlag, 396.

[12] Oksendal B. (2003), Stochastic differential equations. sixth edition, Berlin, SpringerVerlag.

[13] Reid, W. (1972), Riccati differential equations. volume 86 of Mathematics in Science and Engineering, New York and London, Academic Press. 